14. Colombo, G., et al. 1998. Appetite suppression and weight loss after the cannabinoid antagonist SR 141716. Life Sci. 63:L113-L117.

15. Di Marzo, V., et al. 2001. Leptin-regulated endocannabinoids are involved in maintaining food intake. Nature. 410:822-825.

16. Shimada, M., Tritos, N.A., Lowell, B.B., Flier, J.S and Maratos-Flier, E. 1998. Mice lacking melanin-concentrating hormone are hypophagic and lean. Nature. 396:670-674.

17. Qian, S., et al. 2002. Neither agouti-related protein nor neuropeptide $\mathrm{Y}$ is critically required for the regulation of energy homeostasis in mice. Mol. Cell. Biol. 22:5027-5035.

18. Broberger, C., Landry, M., Wong, H., Walsh, J.N., and Hokfelt, T. 1997. Subtypes Y1 and Y2 of the neuropeptide $\mathrm{Y}$ receptor are respectively expressed in pro-opiomelanocortin- and neuropeptide-Y-containing neurons of the rat hypo- thalamic arcuate nucleus. Neuroendocrinology. 66:393-408

19. Broberger, C., Visser, T.J., Kuhar, M.J., and Hok felt, T. 1999. Neuropeptide Y innervation and neuropeptide-Y-Y1-receptor-expressing neurons in the paraventricular hypothalamic nucleus of the mouse. Neuroendocrinology. 70:295-305.

20. Jain, M.R., Horvath, T.L., Kalra, P.S., and Kalra S.P. 2000. Evidence that NPY Y1 receptors are involved in stimulation of feeding by orexins (hypocretins) in sated rats. Regul. Pept. 87:19-24.

21. Chaffer, C.L., and Morris, M.J. 2002. The feeding response to melanin-concentrating hormone is attenuated by antagonism of the NPY Y(1) receptor in the rat. Endocrinology. 143:191-197.

22. Sainsbury, A., Schwarzer, C., Couzens, M., and Herzog, H. 2002. Y2 receptor deletion attenuates the type 2 diabetic syndrome of ob/ob mice. Diabetes. 51:3420-3427.
23. Kushi, A., et al. 1998. Obesity and mild hyperinsulinemia found in neuropeptide Y-Y1 receptordeficient mice. Proc. Natl. Acad. Sci. U. S. A 95:15659-15664.

24. Barsh, G.S., and Schwartz, M.W. 2002. Genetic approaches to studying energy balance: percep tion and integration. Nat. Rev. Genet. 3:589-600.

25. Kalra, S.P., et al. 1999. Interacting appetite regulating pathways in the hypothalamic regulation of body weight. Endocr. Rev. 20:67-100.

26. Cowley, M.A., et al. 2001. Leptin activates anorexigenic POMC neurons through a neural network in arcuate nucleus. Nature. 411:480-484.

27. Cowley, M.A., et al. 2003. The distribution and mechanism of action of ghrelin in the CNS demonstrates a novel hypothalamic circuit regulating energy homeostasis. Neuron. 37:649-661

\title{
Renal fibrosis: not just PAI-1 in the sky
}

\author{
Agnes B. Fogo
}

Department of Pathology, Vanderbilt University Medical Center, Nashville, Tennessee, USA

A delicate balance exists between ECM synthesis and degradation such that interruption of the corresponding pathways results in increased plasminogen activator inhibitor-1 (PAI-1), pathological matrix accumulation, and glomerulosclerosis. A new study (see the related article beginning on page 379) demonstrates that therapy with a mutant PAI-1 increases matrix turnover and reduces glomerulosclerosis by competing with endogenous PAI-1, suggesting therapeutic utility in the treatment of fibrotic renal disease.

J. Clin. Invest. 112:326-328 (2003). doi:10.1172/JCI200319375.

Progressive fibrosis leads to endorgan failure in multiple organs, including heart, lung, liver, and kidney. The process of substitution of functional parenchymal cells by fibrotic tissue has been intensively investigated. Past progress over the last few decades has resulted in remarkable gains in slowing the progression of chronic kidney disease. The major emphasis has been on control of blood pressure, both systemi-

Address correspondence to: Agnes B. Fogo, MCN C3310, Department of Pathology,

Vanderbilt University Medical Center,

Nashville, Tennessee 37232, USA.

Phone: (615) 322-3114; Fax: (615) 343-7023;

E-mail: agnes.fogo@vanderbilt.edu.

Conflict of interest: The author has declared that no conflict of interest exists.

Nonstandard abbreviations used:

plasminogen activator inhibitor-1 (PAI-1);

tissue-type plasminogen activator (t-PA);

urokinase-type plasminogen activator

(u-PA); unilateral ureteral obstruction

(UUO); angiotensin type 1 (AT1);

epithelial-mesenchymal transition (EMT) cally and within the glomerulus. The efficacy of angiotensin I-converting enzyme inhibitors was linked to control of glomerular pressure beyond systemic pressure effects mediated by dilation of the efferent arterioles of the glomerulus (1). However, normalization of glomerular pressure did not completely halt progression, nor could it regress existing fibrosis. Numerous additional factors thus were implicated in progressive renal injury, including modulation of ECM and parenchymal and infiltrating cell interactions. In addition to angiotensin, TGF- $\beta$ has been recognized as a key mediator of renal fibrogenesis. Interestingly, both angiotensin and TGF- $\beta$ induce plasminogen activator inhibitor-1 (PAI-1) (2).

\section{PAl-1 and fibrosis mechanisms}

How might PAI-1 affect fibrosis? PAI-1 is the major inhibitor of tissuetype plasminogen activator ( $t-P A)$ and urokinase-type plasminogen activator (u-PA), which activate plasminogen to yield plasmin, which in turn degrades fibrin. PAI-1 not only inhibits fibrinolysis but also has complex interactions with matrix, promoting net proteolysis (3). t-PA, u-PA, and plasmin can degrade a wide range of ECM proteins; they also activate latent MMPs, in particular MMP-1 and MMP-3, and indirectly activate MMP-2. Models of glomerular sclerosis and/or interstitial fibrosis have been developed in both rats and mice to further examine these mechanisms. Noble and colleagues previously demonstrated that direct manipulation of the plasmin/plasminogen activator system with recombinant t-PA treatment decreased glomerular matrix accumulation in the anti-Thy1 model of glomerular matrix expansion (4). This model does not result in progressive renal damage and shows no attendant interstitial fibrosis but allows in vivo determination of mechanisms of glomerular matrix accumulation. Interstitial fibrosis can be induced in vivo in either rats or mice by unilateral ureteral obstruction (UUO). In contrast to the anti-Thy1 model, UUO does not result in significant glomerular lesions but demonstrates early macrophage infiltration and robust interstitial fibrosis, along with tubular injury. The unobstructed contralateral kidney serves as an ideal control. The UUO model thus allows in-depth in vivo study of mechanisms of interstitial fibrosis, and interactions between epithelial and infiltrating cells. The use of diverse models of renal scarring thus allows 


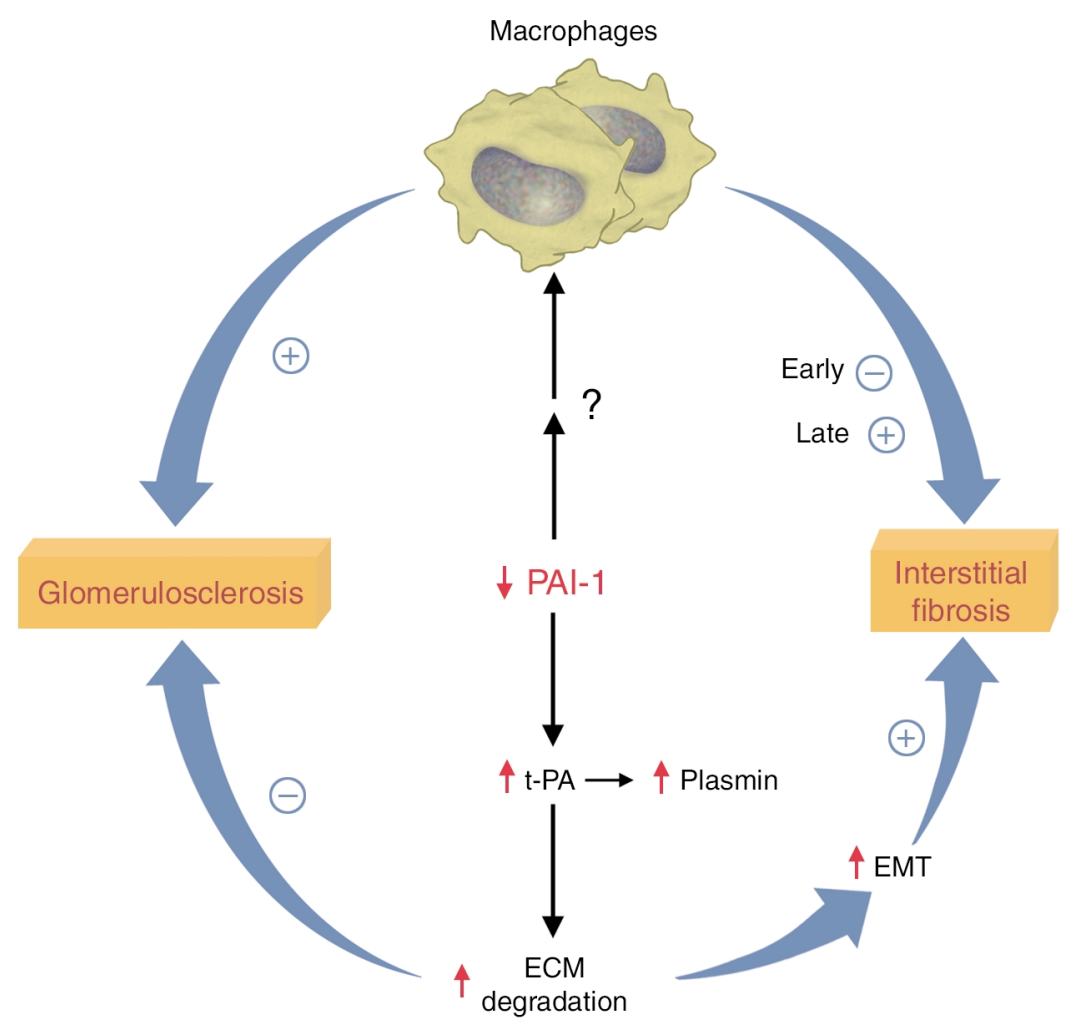

Figure 1

PAI-1 modulates scarring in a complex manner, including effects on cell migration, matrix turnover, and macrophage infiltration. Whereas increased t-PA promotes dissolution of matrix and subsequently lessens glomerulosclerosis, this matrix breakdown facilitates cell migration and epithelial-mesenchymal transition (EMT) and thus contributes to increased interstitial fibrosis. The effects of PAI- 1 that facilitate enhanced macrophage infiltration in vivo are complex and have not yet been established. Macrophages have beneficial effects early in interstitial fibrosis and promote fibrosis later. The increased number of glomerular macrophages is linked to glomerulosclerosis. u-PA and its receptor and vitronectin also interact with PAI-1 and contribute to its final effects on fibrosis.

dissection of the divergent mechanisms that contribute to glomerular versus interstitial scarring (Figure 1). Thus, in contrast to the effects of t-PA on glomerular matrix accumulation, $\mathrm{t}^{-\mathrm{PA}^{-/-}}$mice have reduced interstitial fibrosis after injury caused by UUO (see below) (5).

In normal, non-scarred kidneys, there is very little PAI-1 expression, but acute infusion of angiotensin markedly upregulates PAI-1 mRNA and protein via the angiotensin type 1 (AT1) receptor by a non-pressuredependent mechanism (6). This induction has been shown in vitro to be augmented by aldosterone via a putative glucocorticoid-response element in the PAI-1 promoter (7). In fibrotic renal diseases, PAI-1 is also increased and localizes to areas of glomerulosclerosis (8). Conversely, glomerular plasmin with direct degradation of accumulated excess matrix.

However, the results of Noble and colleagues (11) suggest that PAI-1 also has more far-reaching effects, including indirect effects on matrix synthesis. Thus, mRNA expression for matrix molecules was also decreased, perhaps because of the reduced macrophage infiltration observed with PAI-1 inhibition. The mechanisms for decreased macrophage infiltration with PAI-1 inhibition remain to be determined (Figure 1). The macrophage has a particularly critical role after injury. Macrophages produce numerous profibrotic factors, which increase matrix synthesis by resident parenchymal cells. However, in the absence of TGF- $\beta$ signaling, macrophages may be ineffectual in promoting fibrosis. This has been illustrated experimentally in $\beta_{6}$ integrin knockout mice. The heterodimeric integrin $\alpha_{v} \beta_{6}$, expressed in epithelia in the skin, lung, and kidney, is one key local activator of TGF- $\beta$. TGF- $\beta$ circulates in an inactive form, linked to latencyassociated peptide (LAP). $\beta_{6}$ integrin binds to this TGF- $\beta$-LAP inactive complex, effecting local TGF- $\beta$ activation. $\beta_{6}{ }^{-1-}$ mice were resistant to lung fibrosis induced by bleomycin, despite robust numbers of infiltrating macrophages (12). We have shown similar protection in $\beta_{6}{ }^{-1-}$ mice in the renal fibrosis model of UUO (13). Thus, macrophages may not be profibrotic in the absence of local TGF- $\beta$ activation.

\section{Macrophages and fibrosis}

Of note, glomerular and interstitial macrophage infiltration may not activate identical injury responses. This possibility is supported by our observation of differing phenotype of glomerular versus interstitial macrophages in human diabetic nephropathy; only the latter expressed PPAR- $\gamma$ (14). It is also possible that macrophage infiltration, especially interstitial, has an early, beneficial role after injury, whereas persistent macrophage infiltration, whether glomerular or interstitial, is profibrotic. Recently, we have further demonstrated that infiltrating macrophages 
even play a beneficial role in early injury after UUO, and this salutary effect is dependent on intact AT1 receptor (15). Wild-type mice were radiated and underwent bone marrow reconstitution with $A T 1 a^{-/-}$marrow. Surprisingly, these mice had more severe fibrosis after UUO than identically treated mice reconstituted with wild-type marrow. These beneficial effects of macrophages are similar to the well-established role of macrophages in early wound healing, in which phagocytosis is a key element in resolution of injury. In Noble and colleagues' study (11), macrophage infiltrate was decreased, which could be causal in decreasing glomerular injury. Whether PAI-1 antibody also decreases interstitial macrophages and thereby contributes to decreased interstitial fibrosis remains to be determined. Even those remaining glomerular macrophages could be inhibited in transducing fibrotic effects, because TGF- $\beta$ content of glomeruli was also reduced by the mutant PAI-1, thus reducing downstream fibrosis-effector mechanisms. This decrease in TGF- $\beta$ possibly could result from increased clearance of TGF- $\beta$ by PAI- 1 mutant protein binding to vitronectin.

\section{PAI-1 and cell migration}

PAI-1 also importantly modulates cellular adhesion and migration and thus has impact on inflammation, wound healing, angiogenesis and cell migration, and tumor cell metastasis (3). PAI-1 competes with the u-PA receptor (u-PAR) for the $\mathrm{NH}_{2}$ terminus-binding domain on vitronectin. When PAI-1 binds to vitronectin, u-PAR interaction with the vitronectin and surrounding ECM is prevented, thus inhibiting vitronectin-dependent cellular adhesion and migration. This effect is demonstrated in PAI-1/- mice, which show enhanced smooth muscle cell migration, while PAI- 1 overexpression in vitro inhibits this process $(16,17)$. However, in vivo PAI-1 enhances cell migration in some settings. Increased PAI-1 is linked to macrophage infiltration, and more aggressive tumor cell metastasis, pointing to complex interactions with vitronectin and matrix in vivo, and possible direct chemotactic actions of PAI-1 (Figure 1) (3). The mutant PAI-1 used by Noble and colleagues (11) maintains interactions with vitronectin and thus can have cell migrationinhibitory effects independent of its proteolytic activity. This might not only decrease macrophage infiltration but also affect epithelial-mesenchymal transition (EMT). EMT is characterized by migration of epithelial cells into the interstitium, where they become mesenchymal, myofibroblast-type cells. Neilson and colleagues have recently elegantly demonstrated that this process contributes to renal fibrosis in vivo after UUO (18). The importance of EMT in interstitial fibrosis was also supported by a decrease in interstitial collagen after UUO in $\mathrm{t}-\mathrm{PA}^{-/-}$mice. This protection was linked to decreased MMP-9 induction and preserved tubular basement membrane integrity, postulated to prevent EMT. These findings contrast with the effects of increased t-PA to protect against glomerular matrix accumulation, discussed above. The mutant PAI-1 antibody used by Noble and colleagues could possibly inhibit this migration by its persistent interaction with vitronectin. However, effects on interstitial fibrosis cannot be extrapolated from their studies in the anti-Thy1 model, which has only mesangial proliferation and matrix expansion and no fibrosis.

In conclusion, the studies of Noble and her colleagues (11) show us that modulating fibrosis is a goal within reach, and inhibition of these mechanisms could confer further benefits beyond existing therapies in treatment of chronic kidney diseases. It is important to note that disease was not completely prevented, indicating that additional targets might yet remain for optimal intervention in fibrosis. Further understanding of the complex interactions of parenchymal cells, infiltrating cells, and matrix will allow optimal targeting and development of novel antifibrotic therapies. Synergistic therapies could halt progression of lesions and even potentially regress existing scarring (19).

1. Brenner, B.M. 2002. Remission of renal disease: recounting the challenge, acquiring the goal. J. Clin. Invest. 110:1753-1758. doi:10.1172/ JCI200217531.

2. Ma, L.-J., and Fogo, A.B. 2001. Angiotensin as inducer of plasminogen activator inhibitor-1 and fibrosis. Contrib. Nephrol. 135:161-170.

3. Eddy, A.A. 2002. Plasminogen activator inhibitor-1 and the kidney. Am.J. Physiol. Renal Physiol. 283:F209-F220.

4. Haraguchi, M., Border, W.A., Huang, Y., and Noble, N.A. 2001. t-PA promotes glomerular plasmin generation and matrix degradation in experimental glomerulonephritis. Kidney Int. 59:2146-2155.

5. Yang, J., et al. 2002. Disruption of tissue-type plasminogen activator gene in mice reduces renal interstitial fibrosis in obstructive nephropathy. J. Clin. Invest. 110:1525-1538. doi:10.1172/JCI200216219.

6. Nakamura, S., Nakamura, I., Ma, L., Vaughan, D.E., and Fogo, A.B. 2000. Plasminogen activator inhibitor-1 expression is regulated by the angiotensin type 1 receptor in vivo. Kidney Int. 58:251-259.

7. Brown, N.J., et al. 2000. Aldosterone modulates plasminogen activator inhibitor-1 and glomerulosclerosis in vivo. Kidney Int. 58:1219-1227.

8. Oikawa, T., Freeman, M., Lo, W., Vaughan, D.E., and Fogo, A. 1997. Modulation of plasminogen activator inhibitor- 1 in vivo: a new mechanism for the anti-fibrotic effect of renin-angiotensin inhibition. Kidney Int. 51:164-172.

9. Brown, N.J., et al. 2000. Synergistic effect of adrenal steroids and angiotensin II on plasminogen activator inhibitor-1 production. J. Clin. Endocrinol. Metab. 85:336-344.

10. Oda, T., et al. 2001. PAI-1 deficiency attenuates the fibrogenic response to ureteral obstruction. Kidney Int. 60:587-596.

11. Huang, Y., et al. 2003. A mutant, noninhibitory plasminogen activator inhibitor type 1 decreases matrix accumulation in experimental glomerulonephritis. J. Clin. Invest. 112:379-388. doi:10.1172/JCI200318038.

12. Munger, J.S., et al. 1999. The integrin alpha v beta 6 binds and activates latent TGF beta 1: a mechanism for regulating pulmonary inflammation and fibrosis. Cell. 96:319-328.

13. Ma, L.-J., et al. 2003. Transforming growth factor- $\beta$ dependent and independent pathways of induction of tubulointerstitial fibrosis in $\beta 6^{-/}$ mice. Am. J. Pathol. In press.

14. Paueksakon, P., Revelo, M.P., Ma, L.J., Marcantoni, C., and Fogo, A.B. 2002. Microangiopathic injury and augmented PAI-1 in human diabetic nephropathy. Kidney Int. 61:2142-2148.

15. Nishida, M., et al. 2002. Absence of angiotensin II type 1 receptor in bone marrow-derived cells is detrimental in the evolution of renal fibrosis J. Clin. Invest. 110:1859-1868. doi:10.1172/ JCI200215045.

16. Redmond, E.M., et al. 2001. Endothelial cells inhibit flow-induced smooth muscle cell migration: role of plasminogen activator inhibitor-1. Circulation. 103:597-603.

17. Proia, R.R., et al. 2002. The effect of endothelial cell overexpression of plasminogen activator inhibitor-1 on smooth muscle cell migration. J. Vasc. Surg. 36:164-171.

18. Iwano, M., et al. 2002. Evidence that fibroblasts derive from epithelium during tissue fibrosis. J. Clin. Invest. 110:341-350. doi:10.1172/ JCI200215518.

19. Fogo, A. 2001. Progression and potential regression of glomerulosclerosis. Nephrology Forum. Kidney Int. 59:804-819. 